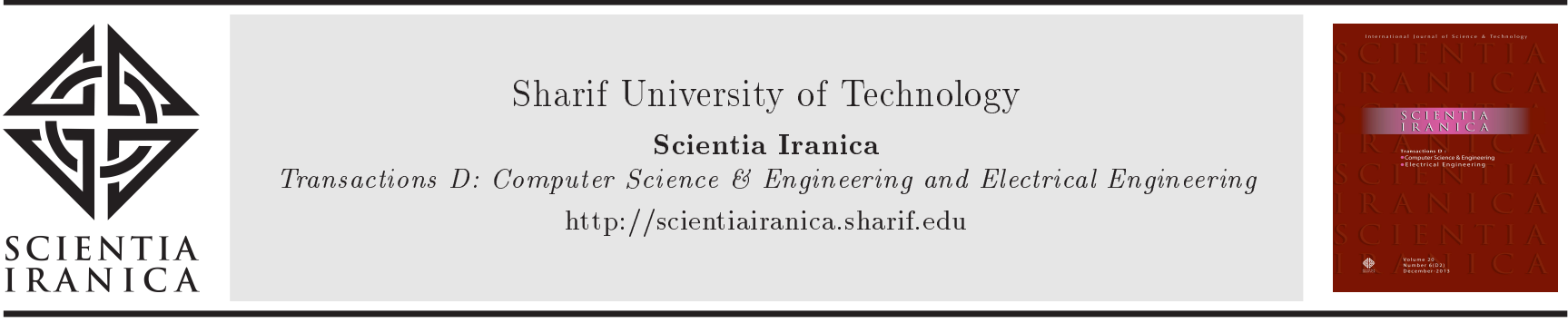

\title{
Generation rejection scheme based on a combinational rotor angle trajectory prediction
}

\author{
A.A. Hajnorouzi ${ }^{a}$, F. Aminifar ${ }^{b}$, and H.A. Shayanfar ${ }^{a, *}$ \\ a. Centre of Excellence for Power System Automation and Operation, Iran University of Science and Technology, Tehran, Iran. \\ b. School of Electrical and Computer Engineering, College of Engineering, University of Tehran, Tehran, Iran. \\ Received 29 July 2019; received in revised form 5 October 2019; accepted 21 December 2019
}

\author{
KEYWORDS \\ Transient stability; \\ Response-based \\ Generation Rejection \\ Scheme (GRS); \\ Phasor Measurement \\ Unit (PMU); \\ Angular stability \\ prediction; \\ Rotor angle trajectory.
}

\begin{abstract}
This paper presents a response-based Generation Rejection Scheme (GRS) based on angular stability prediction logic to initiate the outage of accelerated generating units while saving the rest of generating units from the loss of synchronism. First, trigonometric, polynomial, and hybrid models of rotor angle trajectory based on reasonable assumptions were validated. Then, in the prediction step, through maximum use of measured data based on defined Forecast Horizon (FH) and data window with an incremental length, the stability/instability of generating units was separately predicted. Next, the status of the tripping signal based on the combinational logic of the output results for the angular stability prediction method was determined. In the developed logic, if at least two models out of the three designated models yielded the same response about the unit stability status, the tripping signal was accordingly fired or blocked. The proposed method was employed to investigate the one-machine infinite bus and the WSCC standard test bed under different operations and fault scenarios. The obtained results demonstrated that besides simplicity, low computational burden, and very short processing time, the proposed combinatorial method outperformed the existing ones working with individual prediction models.
\end{abstract}

(C) 2021 Sharif University of Technology. All rights reserved.

\section{Introduction}

Power system always faces a wide variety of disturbances and the main task of protection schemes is to protect power network and equipment against all kinds of events and ensure the correct and continuous operation of a power grid. The importance of the proper performance of the protective system becomes highlighted when the power system is subjected to a large disturbance because it might lose its stability. In case of a blackout, the restoration process would be extremely time-consuming and very costly.

\footnotetext{
*. Corresponding author.

E-mail address: Shayanfar.ha@gmail.com (H.A. Shayanfar)
}

doi: $10.24200 /$ sci. 2019.54136 .3611
Power system protection is categorized into two classes: equipment protection and system protection. The equipment protection mostly focuses on the safety of pieces of equipment in operation. Hence, in the event of any abnormal condition damaging given equipment, the protection activates and isolates the equipment from the network. In contrast, system protection is only attributed to the secure and continuous operation of the system and does not correspond to a single piece of equipment. It may, however, reject some load demands, generators, or other equipment from the system to maintain the rest safely [1]. Special Protection Schemes (SPSs) are a major class of system protection approach. SPSs are often employed as secondary protection schemes [2]. Generator Rejection Schemes (GRSs) are among commonly used SPSs in power system operations [3]. 
GRSs or generator tripping schemes are emergency control systems designed to initiate preplanned outage of one or more accelerated generating units of power plants as prompt corrective actions to shield the rest of generating units against the loss of synchronism [4]. Also, some GRSs may be designed to restore the power balance of the network following a contingency. GRSs are classified into two major groups: offline and online GRS. Offline GRS triggers generator tripping based on offline calculation and a pre-prepared lookup table. However, online GRS which keeps power plants away from the loss of synchronism uses the transient instability prediction approach [5]. Proper operation of both offline and online GRSs is directly dependent upon the accuracy and speed of the decision-making logic. Offline GRS decision process is simple, but its satisfactory performance may likely be subject to serious risk in a real power system with constant changes in load levels and generation patterns. This shortcoming has, to a great extent, been alleviated in the online GRS working with high-resolution data of Phasor Measurement Units (PMUs). PMU as a basis for Wide-Area Monitoring, Protection, And Control (WAMPAC) has a crucial role in the detection and prevention of cascading events such as transient instability of generating units [6]. Such disturbances in the absence of adequate control and protection systems will increase the rotor angle of the generating unit unexpectedly and eventually, synchronization with the network will be lost [7].

Another method for protecting the power plant against transient instability conditions is the use of Out-of-Step (OS) relays. Regarding the overlap between online GRS and OS, it should be emphasized that GRS is predictive and acts before OS to sacrifice some oscillating units in favor of maintaining the others' stability. Therefore, it is designed to reduce the impacts of unit outflows in addition to saving the system and the units' safety. However, in the event of any failure of the GRS, the OS relay still guarantees the system stability.

The trajectory of rotor angle as the most important characteristic of power system transient instability can reflect the response of generating units to severe disturbances. Analysis and process of this feature, before the occurrence of loss of synchronism, would provide invaluable data to initiate the GRS and diminish the intensity of perturbation. Transient instability detection consists of curve-fitting extrapolation techniques. This category predicts the future angular trajectory of generating units based on geometric attributes of the response curve along with predefined threshold value criteria [8]. The result of this process is used to activate GRS in unstable cases or to prevent the operation of GRS in stable events.

Research studies that have investigated SPS from different angles have been reported in the literature. In [9], improvement of transient stability through the enhancement of SPS at Korea electric power system was analyzed. This approach uses a static synchronous compensator (STATCOM) to improve transient stability. In [10], a real-time method based on PMU data was presented to restore stable operation of power systems in case of abnormal conditions. A generator shedding control through a PMU-based model-free method was proposed in [11]. This method predicts the transient stability of generating units using geometrical characteristics of equivalent One-Machine-Infinite-Bus (OMIB)-w curve and definition of new stability criteria. In [12], two online and offline GRSs based on a hybrid method were presented. Both GRSs were used to decrease the risk of an unnecessary outage of generating units. In [4], an analytical approach to determining the safe margins of GRS was developed. To implement this method, a practical framework alongside PMU data and Energy Management System (EMS) was proposed. In [3] and [12], probabilistic methods were employed to decrease the risk of GRS. Both of the referenced studies have used the polynomial method to predict transient instability situations and deploy online GRS to prevent instability of generating units. In [12], the effect of starting point and width of Data Window (DW) designated for transient stability prediction was additionally analyzed. The paper showed that an optimal DW with a proper starting point and DW increased GRS accuracy and security. In [13], for the sake of transient stability prediction, a new set of criteria based on frequency deviation, angle, and magnitude of the load bus voltage as well as the generator angle security were introduced. In [14], a two-stage approach was proposed to predict rotor angle trajectory after a large event. In [15], an adaptive autoregressive method was used to predict the future power angle of a generating unit to estimate the transient behavior of the generator. In [16], based on the concept of center of inertia, two equivalent machines were used to represent coherent areas oscillating against each other. Then, the system trend slope toward uncontrolled separation was predicted by extending the speed-acceleration locus curve. In [17], a new index was proposed to predict angular instability of power systems and islanding prediction. This index is based on the total energy absorbed by generating units in case of islanding. In [18], accuracy and speed of transient stability prediction techniques were investigated. If the output of low-speed or inaccurate methods is used for arming control or protection actions, adverse effects on the reliability and security of the power system will expectedly be inevitable.

This paper presents a response-based GRS by online prediction of power system angular stability to improve the security of power systems in response to 
severe disturbances. The proposed method applies the PMU data measured at the Point of Common Coupling (PCC) of power plant transformer to the bulk power grid. The collected data are used to adjust adaptive DW to predict the angular stability status of the generator in a proper time duration. The proposed method has a combinational logic working with three model predictions which are model-free and based on the mathematical approximation of rotor angle dynamic behavior. The superior features of this method include DW with adaptive width, optimal Forecast Horizon (FH), very low computation burden, non-requirement for equipment models, high accuracy and dependability using new decision logic, and simplicity of application.

Section 2 describes the outline of the proposed methodology. The formulation of the rotor angle trajectory models and their behaviors are presented in Sections 3 and 4, respectively. In Section 5, the new angular stability prediction logic and its performance are discussed. Section 6 concludes the paper.

\section{Outline of the proposed methodology}

The overall configuration of the proposed responsebased GRS is depicted in Figure 1, where the power plant has three similar generating units connected to the external grid via three high-voltage transmission lines. Online GRS has three segments: I) Line Circuit Breaker (CB) status detection: the status of $\mathrm{CB}$ of lines is always monitored by the PMU through its digital input (1 as open and 0 as closed); II) fault detection: it is done based on the online fault detection methods through the PMU input signals; III) angular stability prediction: It is enabled by arming signals of changing the status of CBs and detecting the fault by the PMU. Given that only severe faults near the power plant can lead to angular instability, the CBs of lines connected to the power plant substation are simply monitored. The

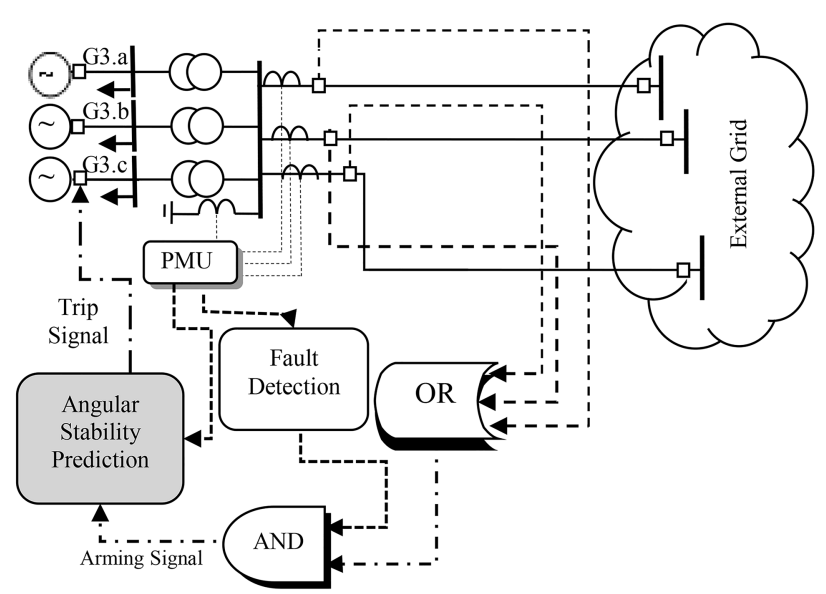

Figure 1. Response-based Generation Rejection Scheme (GRS). arming signal is sent to the stability prediction stage, if both the CB status and fault detection output are equal to one. Therefore, this dual input logic improves the reliability of the method because if each of them is mistakenly activated, GRS function is blocked.

\section{Rotor angle trajectory models}

The first swing of the generating unit's rotor angle following a severe disturbance is either stable or unstable. In the first-swing stable situation, oscillation damps out at the post-fault rotor angle trajectory, while at the unstable trajectory, the rotor angle increases continuously. Much effort has been directed at predicting the future response of the generating unit as the goal of transient stability prediction methods only in the first swing duration $[3,12,19]$. In this context, Single Machine Equivalent (SIME) method is very commonly used to model the rotor angle trajectory.

Section 3 involves the rotor angle trajectory modeling in the following three parts: Trigonometric Model (TM), Polynomial Model (PM), and Hybrid Model (HM).

\subsection{Trigonometric Model (TM)}

The OMIB accelerating power is expressed as $[10,20]$ :

$$
\begin{aligned}
P_{a} & =\frac{W_{0}}{2 H} \frac{d^{2} \delta}{d t^{2}}+D \frac{d \delta}{d t}, \\
P_{a} & =a \delta^{2}+b \delta+c
\end{aligned}
$$

where $P_{a}$ is the accelerating power, $H$ and $D$ are inertia constant and damping factor of the generating unit, respectively, $W_{0}$ is nominal synchronous speed, and $\delta$ is rotor angle value. The temporal characteristic of rotor angle and its first and second derivatives in the time domain are shown below [21]:

$$
\begin{aligned}
& \delta(t)=A \sin \left(w_{d} t+\theta\right), \\
& \frac{d}{d t} \delta(t)=A w_{d} \cos \left(w_{d} t+\theta\right), \\
& \frac{d^{2}}{d t^{2}} \delta(t)=-A w_{d}^{2} \sin \left(w_{d} t+\theta\right),
\end{aligned}
$$

where $w_{d}$ and $\theta$ are angular velocity of machine smallsignal swings and initial rotor angle, respectively. Inserting Eq. (3) in the right-hand side of Eq. (2) and using $\sin ^{2}\left(w_{d} t+\theta\right)=\frac{1}{2}\left(1+\cos \left(2 w_{d}+2 \theta\right)\right)$ yield:

$$
\begin{aligned}
& P_{a}=a A^{2} \sin ^{2}\left(w_{d} t+\theta\right)+b A \sin \left(w_{d} t+\theta\right)+c \\
& P_{a}=a A^{2}\left(\frac{1}{2}\left(1+\cos \left(2 w_{d}+2 \theta\right)\right)+b A \sin \left(w_{d} t+\theta\right)+c .\right.
\end{aligned}
$$

Similarly, the right-hand side of Eq. (1) could be extended as follows: 


$$
P_{a}=-\frac{W_{0}}{2 H} A w_{d}^{2} \sin \left(w_{d} t+\theta\right)+D A w_{d} \cos \left(w_{d} t+\theta\right) .
$$

Equating Eq. (4) with Eq. (5) and simplifying them yield the following:

$$
\begin{gathered}
\left(b+\frac{W_{0}}{2 H} w_{d}^{2}\right) A \sin \left(w_{d} t+\theta\right)=D A w_{d} \cos \left(w_{d} t+\theta\right) \\
-\frac{a A^{2}}{2} \cos \left(2 w_{d}+2 \theta\right)-\frac{a A^{2}}{2}-c .
\end{gathered}
$$

Finally, Eq. (6) could be expressed as the TM assuming $\delta(t)=A \sin \left(w_{d} t+\theta\right)$ :

$$
\delta(t)=A_{2} \cos \left(2 w_{d}+2 \theta\right)+A_{1} \cos \left(w_{d} t+\theta\right)+A_{0},
$$

where:

$$
\begin{aligned}
& A_{2}=-\left(\frac{2 b H+w_{d}^{2}}{2 H} W_{0}\right)\left(\frac{a A^{2}}{2}\right), \\
& A_{1}=\left(\frac{2 b H+w_{d}^{2}}{2 H} W_{0}\right)\left(D A w_{d}\right), \\
& A_{0}=-\left(\frac{2 b H+w_{d}^{2}}{2 H} W_{0}\right)\left(\frac{a A^{2}}{2}+c\right) .
\end{aligned}
$$

\subsection{Polynomial Model (PM)}

In addition to Eqs. (1) and (2), the OMIB accelerating power is:

$$
P_{a}=P_{m}-P_{e},
$$

where:

$$
P_{e}=P_{e}^{\max } \sin (\delta),
$$

where $P_{m}$ and $P_{e}$ are the input mechanical and output electrical power of the generator, respectively. By keeping the first three orders of McLaurin expansion of $\sin (x)$ [21] and inserting Eq. (10) in Eq. (9), we have:

$$
\begin{aligned}
& \sin (x)=x-\frac{x^{3}}{3 !}+\frac{x^{5}}{5 !} \\
& P_{a}=P_{m}-P_{e}=P_{m}-P_{e}^{\max } \sin (\delta), \\
& P_{a}=P_{m}-P_{e}^{\max }\left(\delta-\frac{\delta^{3}}{3 !}+\frac{\delta^{5}}{5 !}\right) .
\end{aligned}
$$

Inserting Eq. (3) in the right-hand side of Eq. (12) and using $w_{d} t+\theta=x$ yield:

$$
\begin{aligned}
P_{a}= & P_{m}-P_{e}^{\max }\left[A \sin (x)-\frac{A^{3} \sin ^{3}(x)}{3 !}\right. \\
& \left.+\frac{A^{5} \sin ^{5}(x)}{5 !}\right] .
\end{aligned}
$$

Considering the trigonometric functions (14), Eq. (13) can be recast as Eq. (15):

$$
\begin{aligned}
& \sin ^{3}(x)=\frac{3}{4} \sin (x)-\frac{1}{4} \sin (3 x), \\
& \sin ^{5}(x)=\frac{5}{8} \sin (x)-\frac{5}{16} \sin (3 x)+\frac{1}{16} \sin (5 x), \\
& P_{a}=P_{m}-P_{e}^{\max }\left[B_{2} \sin (x)+B_{1} \sin (3 x)\right. \\
& \left.\quad+B_{0} \sin (5 x)\right]
\end{aligned}
$$

where:

$$
\begin{aligned}
& B_{2}=\left(A-\frac{3 A^{3}}{4 !}+\frac{A^{5}}{8 \times 4 !}\right), \quad B_{1}=\left(\frac{A^{3}}{4 !}-\frac{A^{5}}{16 \times 4 !}\right), \\
& B_{0}=\left(\frac{A^{5}}{16 \times 5 !}\right) .
\end{aligned}
$$

Inserting Eq. (11) in the right-hand side of Eq. (15) yields:

$$
\begin{aligned}
P_{a}= & P_{m}-P_{e}^{\max }\left(B_{2}\left(x-\frac{x^{3}}{3 !}+\frac{x^{5}}{5 !}\right)\right. \\
& +B_{1}\left(3 x-\frac{3^{3} x^{3}}{3 !}+\frac{3^{5} x^{5}}{5 !}\right) \\
& \left.+B_{0}\left(5 x-\frac{5^{3} x^{3}}{3 !}+\frac{5^{5} x^{5}}{5 !}\right)\right), \\
P_{a}= & P_{m}-P_{e}^{\max }\left[C_{2} x^{5}+C_{1} x^{3}+C_{0} x\right],
\end{aligned}
$$

where:

$$
\begin{aligned}
& C_{2}=\left(\frac{B_{2}}{5 !}+\frac{3^{5}}{5 !} B_{1}+\frac{5^{5}}{5 !} B_{0}\right), \\
& C_{1}=\left(-\frac{B_{2}}{3 !}+\frac{3^{3}}{3 !} B_{1}+\frac{5^{3}}{3 !} B_{0}\right), \\
& C_{0}=\left(B_{2}+3 B_{1}+5 B_{0}\right) .
\end{aligned}
$$

Eq. (17) with Eq. (5) and simplifying it lead to:

$$
\begin{gathered}
-\frac{W_{0}}{2 H} A w_{d}^{2} \sin \left(w_{d} t+\theta\right)+D A w_{d} \cos \left(w_{d} t+\theta\right) \\
=P_{m}-P_{e}^{\max }\left[C_{2} x^{5}+C_{1} x^{3}+C_{0} x\right] .
\end{gathered}
$$

Keeping the first three orders of McLaurin expansion, we have:

$$
\cos (x)=x-\frac{x^{2}}{2 !}+\frac{x^{4}}{4 !} .
$$

Inserting Eq. (20) in the left-hand side of Eq. (19) and using $w_{d} t+\theta=x$ give: 


$$
\begin{aligned}
A \sin \left(w_{d} t+\theta\right) & =-\frac{2 H}{W_{0} w_{d}^{2}}\left(P_{m}-P_{e}^{\max }\left[C_{2}\left(w_{d} t+\theta\right)^{5}\right.\right. \\
& \left.+C_{1}\left(w_{d} t+\theta\right)^{3}+C_{0}\left(w_{d} t+\theta\right)\right] \\
& -D A w_{d}\left[\left(w_{d} t+\theta\right)-\frac{\left(w_{d} t+\theta\right)^{2}}{2 !}\right. \\
& \left.\left.+\frac{\left(w_{d} t+\theta\right)^{4}}{4 !}\right]\right) .
\end{aligned}
$$

Finally, after the simplification, a five-order PM can be used to predict the rotor angle value, that is:

$$
\delta(t)=D_{5} t^{5}+D_{4} t^{4}+D_{3} t^{3}+D_{2} t^{2}+D_{1} t^{1}+D_{0} .
$$

\subsection{Hybrid Model (HM)}

By inserting Eq. (12) in Eq. (1), the second derivative of rotor angle can be expressed as Eq. (23):

$$
\frac{d^{2} \delta}{d t^{2}}=\frac{2 H}{W_{0}}\left(P_{m}-P_{e}{ }^{\max } \sin (\delta)-D \frac{d \delta}{d t}\right)
$$

Replacing the first derivative of rotor angle with Eq. (3) and $\sin (\delta)$ by Eq. (11) in the right-hand side of Eq. (23) yields Eq. (24):

$$
\begin{aligned}
\frac{d^{2} \delta}{d t^{2}}= & \frac{2 H}{W_{0}} P_{m}-\frac{2 H}{W_{0}}\left(P_{e}{ }^{\max }\left(\delta(t)-\frac{\delta(t)^{3}}{3 !}+\frac{\delta(t)^{5}}{5 !}\right)\right. \\
& \left.-D A w_{d} \cos \left(w_{d} t+\theta\right)\right) .
\end{aligned}
$$

Inserting Eq. (3) in the right-hand side of Eq. (24) using $x=w_{d} t+\theta$, we have:

$$
\begin{aligned}
\frac{d^{2} \delta}{d t^{2}}= & \frac{2 H}{W_{0}} P_{m}-\frac{2 H}{W_{0}}\left(P _ { e } { } ^ { \operatorname { m a x } } \left(A \sin (x)-\frac{A^{3}}{3 !} \sin (x)^{3}\right.\right. \\
& \left.\left.+\frac{A^{5}}{5 !} \sin (x)^{5}\right)-D A w_{d} \cos \left(w_{d} t+\theta\right)\right) .
\end{aligned}
$$

With Eq. (14), Eq. (25) can be rewritten as follows:

$$
\begin{aligned}
\frac{d^{2} \delta}{d t^{2}} & =E_{3} \sin (5 x)+E_{2} \sin (3 x)+E_{1} \sin (x)+E_{0} \\
& -D A w_{d} \cos \left(w_{d} t+\theta\right),
\end{aligned}
$$

where:

$$
\begin{aligned}
& E_{3}=-\frac{A^{5}}{5 ! \times 16} \frac{2 H}{W_{0}} P_{e}^{\max }, \\
& E_{2}=-\frac{2 H}{W_{0}}\left(\frac{A^{3}}{4 !}-\frac{A^{5}}{16 \times 4 !}\right) P_{e}^{\max }, \\
& E_{1}=-\frac{2 H}{W_{0}}\left(A-\frac{A^{3}}{4 \times 2 !}+\frac{A^{5}}{8 \times 4 !}\right) P_{e}^{\max }, \\
& E_{0}=\frac{2 H}{W_{0}} P_{m} .
\end{aligned}
$$

Replacing $x$ and twice integration of Eq. (26), we have:

$$
\begin{aligned}
\frac{d^{2} \delta}{d t^{2}}= & E_{3} \sin \left(5 w_{d} t+5 \theta\right)+E_{2} \sin \left(3 w_{d} t+3 \theta\right) \\
& +E_{1} \sin \left(w_{d} t+\theta\right)+E_{0}-D A w_{d} \cos \left(w_{d} t+\theta\right), \\
\frac{d \delta}{d t}= & -\frac{E_{3}}{5 w_{d}} \cos \left(5 w_{d} t+5 \theta\right)-\frac{E_{2}}{3 w_{d}} \cos \left(3 w_{d} t+3 \theta\right) \\
& -\frac{E_{1}}{w_{d}} \cos \left(w_{d} t+\theta\right)+E_{0} t+F_{1}-D A \sin \left(w_{d} t+\theta\right), \\
\delta(t)= & -\frac{E_{3}}{25 w_{d}^{2}} \sin \left(5 w_{d} t+5 \theta\right)-\frac{E_{2}}{9 w_{d}^{2}} \sin \left(3 w_{d} t+3 \theta\right) \\
& -\frac{E_{1}}{w_{d}^{2}} \sin \left(w_{d} t+\theta\right)+\frac{E_{0}}{2} t^{2}+F_{1} t+F_{0} \\
& +\frac{D A}{w_{d}} \cos \left(w_{d} t+\theta\right) .
\end{aligned}
$$

Finally, with coefficients of Eq. (29), Eq. (28) as a HM can be rewritten as Eq. (30):

$$
\begin{aligned}
F_{2}= & \frac{E_{0}}{2}, \quad G_{3}=-\frac{E_{3}}{25 w_{d}^{2}}, \quad G_{2}=-\frac{E_{2}}{9 w_{d}^{2}}, \\
G_{1}= & -\frac{E_{1}}{w_{d}^{2}}, \quad G_{0}=\frac{D A}{w_{d}}, \\
\delta(t)= & \frac{E_{0}}{2} t^{2}+F_{1} t+F_{0}+G_{3} \sin \left(5 w_{d} t+5 \theta\right) \\
& +G_{2} \sin \left(3 w_{d} t+3 \theta\right)+G_{1} \sin \left(w_{d} t+\theta\right) \\
& +G_{0} \cos \left(w_{d} t+\theta\right) .
\end{aligned}
$$

The three obtained models in Eqs. (7), (22), and (30) as the final models will be used for predicting the rotor angle trajectory (focusing on the first swing) in this paper.

\section{Prediction of angular stability}

Swing equation can be used to compute the rotor angle value as the main data point for the transient stability prediction [3,5]. The main challenge for the mentioned methods is determining the initial value of the parameters in this equation including inertia constant and rotor angle, in addition to inertia variations based on changes in physical and geometrical structure of the generating unit $[3,22]$. To overcome this difficulty, we assume that the generator rotor angle can be estimated based on the voltage phase angle. This assumption has been formulated from the fact that these two mentioned variables, although not equal, have a very similar 
variation trend in transient stability conditions [12]. To access the online value of bus phase angle and the designated DW for the prediction of angular stability, the PMU data synchronized with GPS can be used.

In the following, Section 4.1 describes the DW and FH parameters. Rotor angle stability/instability criteria are explained in Section 4.2. In Section 4.3, the main steps of the rotor angle trajectory prediction for generating units are expressed. In this sub-section, the models introduced in Section 3 are independently used in the rotor angle prediction process. In Section 4.4, the validity and accuracy of models in modeling and predicting the rotor angle trajectory are independently investigated and ultimately compared with each other. The final combinatorial GRS method will be presented in the next section.

\section{1. $D W$ and $F H$}

The time series prediction allows forecasting future values of an observed time series based on the designated DW over a specified FH. The DW has been formed based on the previously measured/gathered data and what is projected in the future represents the FH. To the best of our knowledge, a few studies have discussed the role of DW in predicting the generating unit's rotor angle trajectory. In [12], it was shown that the DW had a considerable effect on the accuracy of the rotor angle prediction method. Therefore, it was proposed that the optimal DW be selected for each system associated with its characteristics. Referring to the practical and research experiences, there was no optimal $\mathrm{FH}$; however, the error of the prediction method can be reduced by selecting an appropriate $\mathrm{FH}$.

The purpose of this paper is to design a responsebased GRS using rotor angle trajectory prediction. The main function of this structure is to prevent system instability by rejecting one or minimum number of generating units after the power system is subjected to a large disturbance. In this regard, the whole process is categorized into the following time periods:

- Data gathering interval $\left(T_{D W}\right)$ : The length of DW specified based on the enough number of PMU phase angle samples;

- Processing interval $\left(T_{\text {Pro }}\right)$ : The time required by the CPU for performing prediction methods;

- Action interval $\left(T_{A c t}\right)$ : The time associated with the action of Generator CB (GCB) to disconnect the unit from the grid.

In previous studies $[3,5]$, the length of DW is constant and equal to $100 \mathrm{~ms}$ after the fault clearance. The Fixed DW (FDW) is used to predict the rotor angle in the future until reaching stability/instability criteria (regardless of any specific FH). This assumption makes the result of the prediction ready much earlier than

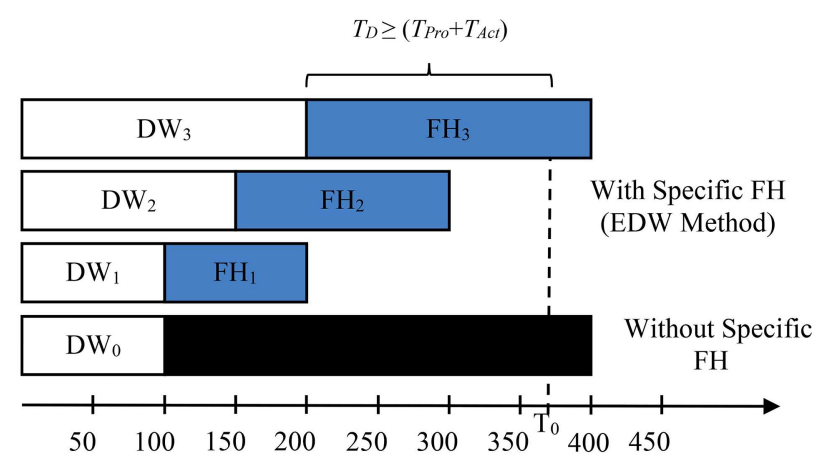

Figure 2. Data Window (DW) and Forecast Horizon (FH) of Fixed DW (FDW) and Extending DW (EDW) methods.

the time needed for the activation of GCB, but the prediction accuracy is sacrificed for the sake of speed. This approach does not make sense in many cases since we can exploit longer DW to reach accurate results, still within a workable time period, of course.

Given that $T_{\text {Pro }}$ is negligible and constant and $T_{A c t}$ has a specific range according to manufacturers' GCB data, adaptive DW is considered here. First, a constant $100 \mathrm{~ms}$ DW is considered and then, according to the comparison of predicted results in the specified $\mathrm{FH}$ with stability criteria, if none of the stability criteria is met, the decision will then be postponed to the next step of prediction by extending the length of DW to $50 \mathrm{~ms}$. This method is known as Extending DW (EDW) in this paper. In line with the general logic of forecasting methods, $\mathrm{FH}$ is assumed to be limited and equal to the DW length at each step of the prediction.

Executive procedures of FDW and EDW methods are displayed in Figure 2. In this figure, $T_{0}$ is the time of determining the first swing behavior in actual data. At this time, one of the two criteria for stability or instability has been met. Unlike the FDW method, the stability/instability result of EDW methods may be presented in the first, second, third, or subsequent DWs. Based on the ultimate goal of the GRS in unstable situations, $T_{D}$ as the time interval between the data gathering step (the end of the DW) and time of real stability/instability is equal to or more than $T_{\text {Pro }}+T_{\text {Act }}$.

\subsection{Rotor angle stability/instability criteria}

There are some criteria for detecting instability using future angle trajectories. In the present work, stability/instability criteria proposed in [3] and [5] are used to determine the stability of the generating unit. Accordingly, a power plant is going to be unstable if the slop sign of the phase angle is always positive and the relative phase angle with respect to the reference bus passes the boundary value of the stability angle. The instability limit of the rotor angle trajectory is assumed to be 180 degrees $[3,5,12,23]$. Positive and negative 
signs of phase angle reflect the growth of rotor angle after fault clearance and its decline before crossing the stability boundary. In this case, the generator will remain stable.

\subsection{Rotor angle trajectory prediction for generating units}

Figure 3 depicts the three main steps of applying the EDW method based on the independent application of each rotor angle stability prediction model for generating units (PM/TM/HM). The figure is explained in the following:

Step 1. Fault detection: The first step is to identify a possible fault and its clearance time to begin the rotor angle prediction step;

Step 2. DW Preparation and curve-fitting: Calculation of DW starts with the initial data after fault clearance and with the length of $L \mathrm{~ms}$. The

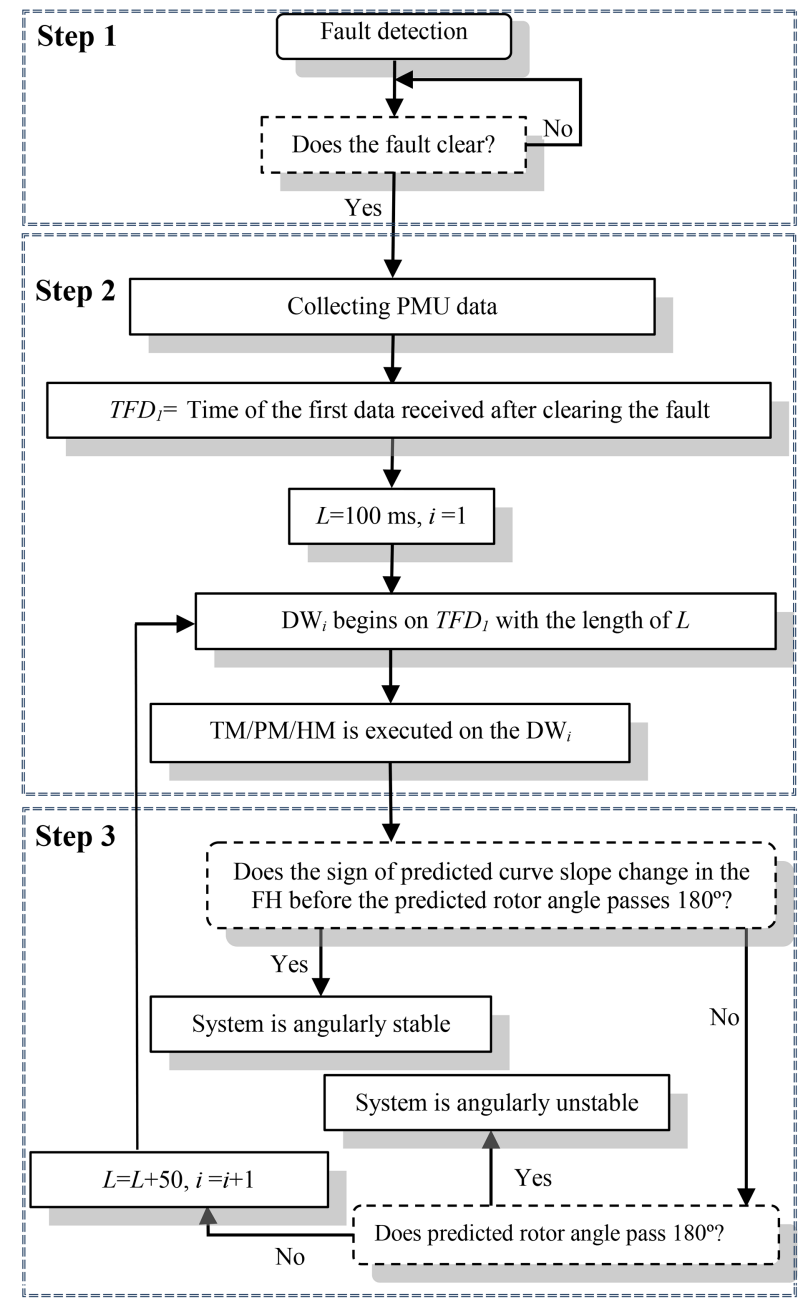

Figure 3. Flowchart of the Extending DW (EDW) method based on the independent application of each rotor angle stability prediction model for generating units (PM/TM/HM). initial value of $L$ is $100 \mathrm{~ms}$ which may increase in the next prediction steps to $50 \mathrm{~ms}$ upon each iteration. The voltage phase angle measured by PMU on the high-voltage side of the power plant transformer is the DW input data. When DW is filled out, each of the aforementioned models (TM/PM/HM) is individually executed and three resulting curve fittings are obtained;

Step 3. Decision making: For each model, based on the result of Step 2, first, the slope of the resulting curve is determined. In the next part, based on the slope sign at the $\mathrm{FH}$, three conditions may occur for the first swing: I) stable case: In this case, for one point of the predicted curve in $\mathrm{FH}$, the sign of slope changes from positive to negative and the corresponding rotor angle is less than 180 degrees; II) unstable case: When slop sign is always positive and the rotor angle in FH constantly grows and finally passes the 180 degrees; and III) unknown case: When none of the stability/instability criteria is met in the FH. For example, slope sign is always positive, but the rotor angle in $\mathrm{FH}$ is less than 180 degrees. In this case, decision-making will be postponed to the next step of prediction by extending the length of DW to $50 \mathrm{~ms}$.

\subsection{Rotor angle model validation}

The proposed methodology is applied to the SIME and WSCC 9-bus test systems, as shown in Figure 4. SIME consists of $128 \mathrm{MVA}$ and $115 \mathrm{MW}$ generating units which are connected to the $400 \mathrm{kV}$ substation through one $12.7 / 400 \mathrm{kV}$ transformer. WSCC 9-bus incorporates a reference generator (G1-salient pole) and two power plants including generators in the $\mathrm{PV}$ mode (G2 and G3 round rotors). The G2 power plant consists of three identical units called G2.a, G2.b, and G2.c. All generators have an IEEE DC1 excitation system and a full-order model. A PMU device with a sampling rate of $10 \mathrm{kHz}$ and a reporting rate of $100 \mathrm{sample} / \mathrm{s}$ is installed on the high-voltage side of the substation. The input data of the PMU is supplied through current and voltage transformers $(\mathrm{CT}, \mathrm{VT})$. For the sake of time-domain simulation, the DIgSILENT software package is used. Curve fitting and decision-making process are performed in the MATLAB environment.

To validate the accuracy of the three models (TM/PM/HM) described in Section 3, two case studies including marginally stable and marginally unstable ones are simulated on SIMB test system. The Critical Clearing Time (CCT) of the three-phase-to-ground (LLLG) fault occurring at $t=0$ and $0.1 \%$ of Line 1 (L1) of SIMB is $0.329 \mathrm{sec}$. Thus, to simulate the aforementioned case studies, the fault is cleared after $t=0.328$ and $0.330 \mathrm{sec}$, respectively. Based on the simulation results and their comparison with stability 


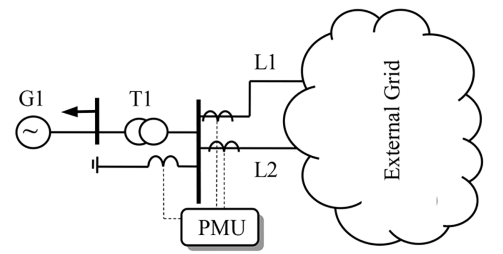

(a) SIME

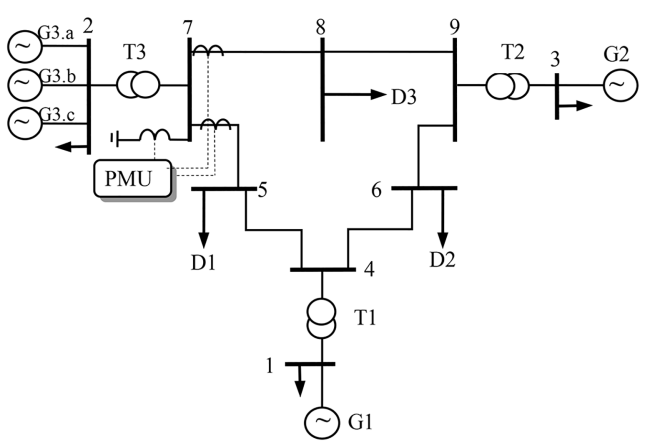

(b) WSCC 9-bus

Figure 4. Single-line diagram of test systems.

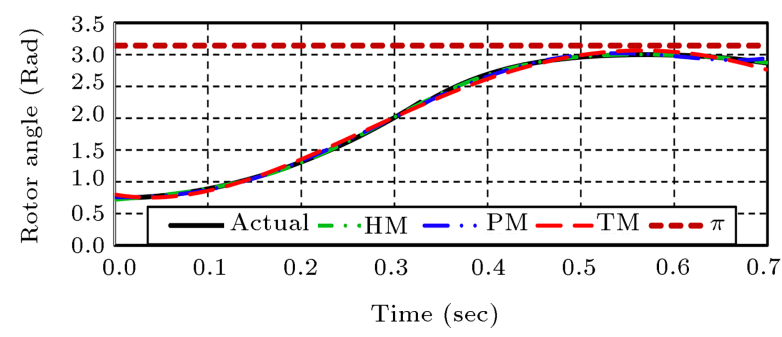

(a) SIME-marginally stable case

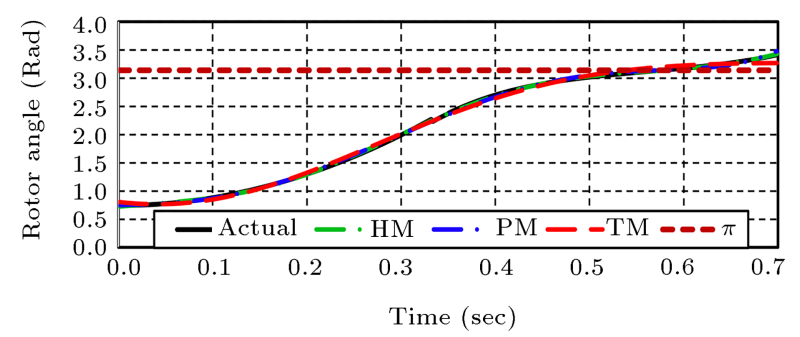

(b) SIME-marginally unstable case

Figure 5. Rotor angle modeling results based on the proposed rotor angle prediction models for Single Machine Equivalent (SIME), three-phase-to-ground (LLLG) fault, $0.1 \%$ of L1.

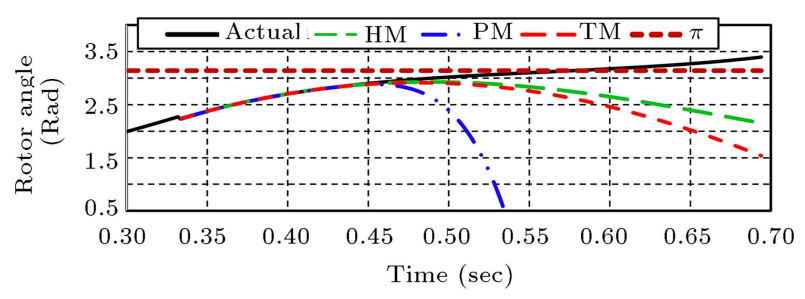

(a) SIME-marginally unstable case-FDW method

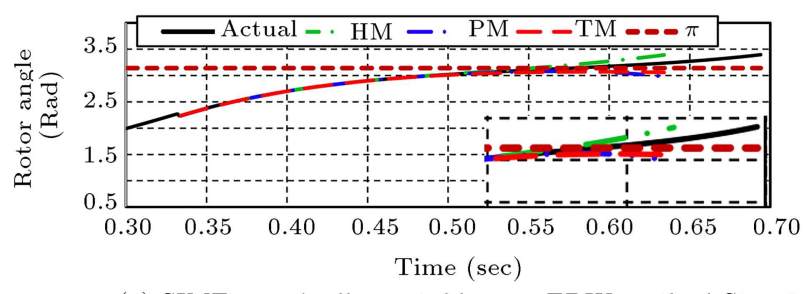

(c) SIME-marginally unstable case-EDW method-Step 2

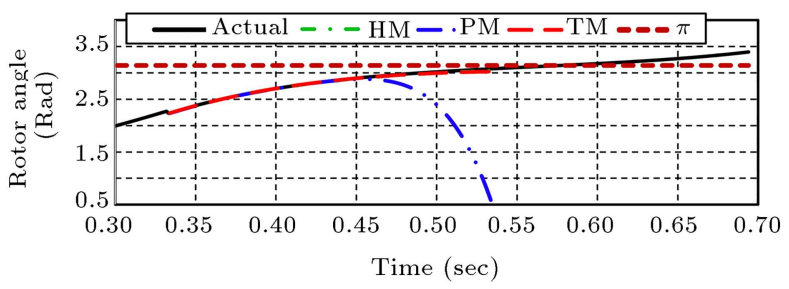

(b) SIME-marginally unstable case-EDW method-Step 1

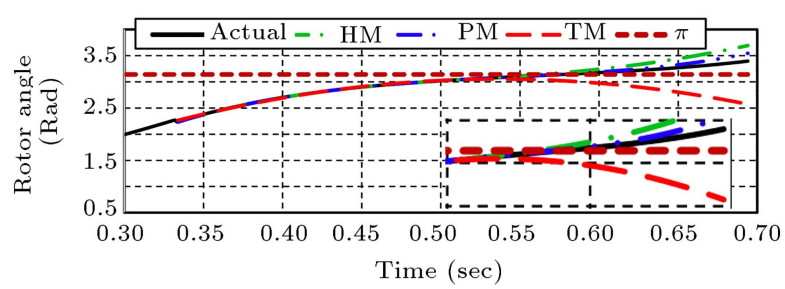

(d) SIME-marginally unstable case-EDW method-Step 3

Figure 6. Rotor angle prediction results based on Fixed DW (FDW) method (without FH) and Extending DW (EDW) method (with FH) for Single Machine Equivalent (SIME) and three-phase-to-ground (LLLG) fault at $0.1 \%$ of L1.

margin $\left(180^{\circ}\right)$ shown in Figure 5, all models (TM, PM, and HM) accurately followed the rotor angle trajectory in both cases. Similar simulations for different system loading levels, fault locations, and fault durations were performed and the results proved the accuracy and validity of models, especially for the first swing.

\subsection{Model prediction performance}

The enhancement of prediction responses for EDW method versus FDW method is demonstrated in Fig- ure 6. Figure 6(a) shows a valuable observation about the system angular stability prediction based on the FDW method. In this case, the actual bus phase angle is marginally unstable, while the results of all the three predictions are incorrectly stable. In Figure 6(b) to (d), the following results are obtained through the application of the EDW method. In Figure 6(b), in Step $1(\mathrm{DW}=\mathrm{FH}=100 \mathrm{~ms}), \mathrm{PM}$ predicts the stability of the system; however, in TM and HM, none of the instability and stability conditions are satisfied (the 
behavior of all three methods at the end of $\mathrm{FH}$ is shown in the zoomed part of the figures). In Figure 6(c), HM predicts angular instability in Step $2(\mathrm{DW}=\mathrm{FH}=$ $150 \mathrm{~ms}$ ); however, TM and PM do not have explicit predictions. In Step $3(\mathrm{DW}=\mathrm{FH}=200 \mathrm{~ms})$, according to Figure 6(d), HM and PM correctly predict the angular instability and TM has an opposite prediction.

To evaluate the performance of the proposed models, they were tested separately in the framework of the FDW and EDW methods in different case studies of WSCC 9-bus test system. To investigate the effect of fault location and generation level of G2 power plant, the LLLG fault with $0.8 \Omega$ resistance and $120 \mathrm{~ms}$ duration is applied on Lines 7-5 and 7-8. Fault is cleared by opening the line $\mathrm{CB}$. At each generation level of $\mathrm{G} 2$, the fault location is moved from beginning to end of the faulted line with $1 \%$ steps of its length.

Table 1 shows the summarized results of Line 7 8. Columns 2 to 4 and 5 to 7 show prediction errors of FDW and EDW methods in stability/instability situations in the aforementioned case studies, respectively. The numbers in parentheses in Columns 5 to 7 denote the steps for obtaining the result at the specified generation level of G2 and the fault location with the longest input DW. The accuracy of fault prediction on Line 7-5 is also shown in Table 2. For the situation with the highest stress (high generation level), results showed that EDW method outperformed FDW method. In other words, prediction results of PM, TM, and HM improved by $10 \%, 3 \%$, and $2 \%$ at high generation levels on Line 7-8, respectively. According to Table 2, the performance of EDW method improved by $25 \%$ and $8 \%$ for $\mathrm{PM}$ and TM, respectively, in the best situation (PG2 $=150 \mathrm{MW}$ ) and $8 \%$ for $\mathrm{HM}$ in
PG2 = $147 \mathrm{MW}$. It can be concluded that in general, $\mathrm{PM}$ correctly predicts the entire system instability situation, although it fails in case of marginal stability. TM runs into error under instability and correctly predicts system stability while HM may face error in stable or unstable conditions. However, HM achieves a more accurate prediction in case of marginally stable or marginally unstable conditions and it is applied as a means to correct the erroneous performance of TM and PM in the proposed GRS. Regarding the parameter estimation of models, there are more parameters associated with HM to be estimated. However, it is not challenging since the problem is still overdetermined. That is, the number of unknown parameters is less than the minimum number of data points in DW (10 points).

By adopting the instability limit less than $180^{\circ}$, in case of a marginally stable condition (as illustrated in Figure 5(a)), the GRS sends a false tripping signal to generating units, although the power plant remains stable. Therefore, the performance accuracy of the GRS is overlooked here.

Based on the methods outlined in [3] and [12], further comparisons are made to investigate the performance of the proposed models. In this case, the lengths of DW1, DW2, and DW3 are 80, 100, and $120 \mathrm{~ms}$, respectively. The generation level of G2 ranges from 148 to $155 \mathrm{MW}$. Based on the location of the fault on Line $7-8$ and the generation level of G2, the sum of the simulated states is equal to 400 cases. Table 3 shows the summarized results of this comparison. Results demonstrated that EDW outperformed FDW method (with a different rotor angle trajectory model and DW). For FDW method, the length of DW is fixed between 80-120 ms, although the DW length for the EDW

Table 1. Prediction error of Trigonometric Model (TM), Polynomial Model (PM), and Hybrid Model (HM) for the three-phase-to-ground (LLLG) fault on Line 7-8.

\begin{tabular}{|c|c|c|c|c|c|c|}
\hline \multirow[b]{4}{*}{$\begin{array}{c}\text { Generation level of } \\
\text { G2 }(\mathrm{MW})\end{array}$} & \multicolumn{6}{|c|}{ LLLG fault } \\
\hline & \multicolumn{6}{|c|}{ Fault time duration $(120 \mathrm{~ms})$} \\
\hline & \multicolumn{3}{|c|}{ FDW } & \multicolumn{3}{|c|}{ EDW } \\
\hline & TM & PM & HM & TM & $\mathbf{P M}$ & HM \\
\hline 155 & 8 & 15 & 7 & $5(2)$ & $3(3)$ & $5(2)$ \\
\hline 154 & 6 & 12 & 5 & $3(2)$ & $3(3)$ & $3(2)$ \\
\hline 153 & 4 & 10 & 4 & $2(2)$ & $3(3)$ & $2(2)$ \\
\hline 152 & 3 & 8 & 2 & $0(1)$ & $3(2)$ & $0(2)$ \\
\hline 151 & 1 & 7 & 0 & $0(1)$ & $2(2)$ & $0(1)$ \\
\hline 150 & 0 & 4 & 0 & $0(1)$ & $1(2)$ & $0(1)$ \\
\hline 149 & 0 & 2 & 0 & $0(1)$ & $0(1)$ & $0(1)$ \\
\hline 148 & 0 & 0 & 0 & $0(1)$ & $0(1)$ & $0(1)$ \\
\hline
\end{tabular}


Table 2. Prediction error of Trigonometric Model (TM), Polynomial Model (PM), and Hybrid Model (HM) for the three-phase-to-ground (LLLG) fault on Line 7-5.

\begin{tabular}{|c|c|c|c|c|c|c|}
\hline \multirow[b]{4}{*}{$\begin{array}{c}\text { Generation level of } \\
\text { G2 (MW) }\end{array}$} & \multicolumn{6}{|c|}{ LLLG fault } \\
\hline & \multicolumn{6}{|c|}{ Fault time duration $(120 \mathrm{~ms})$} \\
\hline & \multicolumn{3}{|c|}{ FDW } & \multicolumn{3}{|c|}{ EDW } \\
\hline & TM & $\mathbf{P M}$ & HM & TM & $\mathbf{P M}$ & HM \\
\hline 155 & 21 & 41 & 22 & $15(2)$ & $18(3)$ & $16(2)$ \\
\hline 154 & 19 & 39 & 20 & $12(2)$ & $16(3)$ & $11(2)$ \\
\hline 153 & 19 & 38 & 18 & $11(2)$ & $15(3)$ & $10(2)$ \\
\hline 152 & 17 & 36 & 15 & $10(1)$ & $15(3)$ & $11(2)$ \\
\hline 151 & 15 & 36 & 14 & $8(2)$ & $12(3)$ & $7(2)$ \\
\hline 150 & 14 & 35 & 12 & $6(2)$ & $10(3)$ & $5(2)$ \\
\hline 149 & 11 & 31 & 12 & $6(2)$ & $9(3)$ & $4(2)$ \\
\hline 148 & 10 & 27 & 9 & $4(2)$ & $8(3)$ & $2(2)$ \\
\hline 147 & 9 & 26 & 9 & $3(2)$ & $8(3)$ & $1(2)$ \\
\hline 146 & 9 & 23 & 8 & $1(2)$ & $6(3)$ & $0(2)$ \\
\hline 145 & 7 & 21 & 6 & $0(2)$ & $5(2)$ & $0(2)$ \\
\hline 144 & 6 & 21 & 6 & $0(2)$ & $4(2)$ & $0(2)$ \\
\hline 143 & 4 & 19 & 5 & $0(2)$ & $2(2)$ & $0(1)$ \\
\hline 142 & 3 & 18 & 3 & $0(1)$ & $1(2)$ & $0(1)$ \\
\hline 141 & 1 & 17 & 0 & $0(1)$ & $1(2)$ & $0(1)$ \\
\hline 140 & 0 & 15 & 0 & $0(1)$ & $0(2)$ & $0(1)$ \\
\hline 135 & 0 & 7 & 0 & $0(1)$ & $0(1)$ & $0(1)$ \\
\hline 130 & 0 & 0 & 0 & $0(1)$ & $0(1)$ & $0(1)$ \\
\hline
\end{tabular}

Table 3. Accumulation prediction error using different data windows and rotor angle trajectory models for the three-phase-to-ground (LLLG) fault on Line 7-8.

\begin{tabular}{|c|c|c|c|c|}
\hline \multirow[b]{4}{*}{ Prediction method } & \multicolumn{4}{|c|}{ LLLG fault } \\
\hline & \multicolumn{4}{|c|}{ Fault time duration $(120 \mathrm{~ms})$} \\
\hline & \multicolumn{3}{|c|}{ FDW } & EDW \\
\hline & DW1 & DW2 & DW3 & DW2 (as first step DW) \\
\hline $\mathrm{TM}$ & 38 & 22 & 32 & $10(2)$ \\
\hline $\mathrm{PM}$ & 45 & 58 & 34 & $17(2)$ \\
\hline HM & 36 & 18 & 27 & $10(2)$ \\
\hline 4th-degree polynomial [3] & 90 & 46 & 56 & $23(2)$ \\
\hline
\end{tabular}

method is $150 \mathrm{~ms}$ in the longest case. Finally, the minimum value of $T_{D}$ for the simulation cases is 75 $\mathrm{ms}$, which is greater than $T_{A c t}$ of $G C B$. $T_{\text {Pro }}$ of all methods is less than $1 \mathrm{~ms}$.

\section{Online generation rejection scheme (GRS)}

Based on Figure 3, the results of the prediction models in EDW method presented at each stage can be classified into three sections: stability (S), instability (IN), or unknown (UN). Having determined these results at each stage, if at least two models have the same output which is not UN, stability/instability of the generating unit is predicted. Otherwise, all three models will be implemented once again by extending the length of DW to $50 \mathrm{~ms}$. In this structure, the feedback path is removed from Figure 3 and embedded in Figure 7. Finally, in case of instability prediction, the tripping signal is fired by the angular stability prediction of GRS and one or more generating units (preplanned based on the offline analysis and power plant generation level) are rejected.

Table 4 shows the performance of the proposed GRS in comparing FDW and EDW methods for LLLG 
Table 4. Comparison of Fixed DW (FDW), Extending DW (EDW), and proposed Generation Rejection Scheme (GRS) responses for WSCC and LLLG fault at $6 \%$ of Line $5-7$ at PG $2=145 \mathrm{MW}$.

\begin{tabular}{|c|c|c|c|c|c|c|c|c|}
\hline \multirow[b]{4}{*}{ DW (ms) } & \multicolumn{8}{|c|}{ LLLG fault } \\
\hline & \multicolumn{8}{|c|}{ Fault time duration $(120 \mathrm{~ms})$} \\
\hline & \multicolumn{3}{|c|}{ FDW } & \multicolumn{3}{|c|}{ EDW } & \multirow[t]{2}{*}{ Proposed GRS } & \multirow[t]{2}{*}{ Actual status } \\
\hline & TM & PM & HM & TM & $\mathbf{P M}$ & HM & & \\
\hline 100 & IN & IN & IN & $\mathrm{S}$ & $\mathrm{UN}$ & $\mathrm{UN}$ & $\mathrm{UN}$ & Marginally stable \\
\hline 150 & - & - & - & $\mathrm{S}$ & IN & $\mathrm{S}$ & S (Block tripping signal) & \\
\hline
\end{tabular}

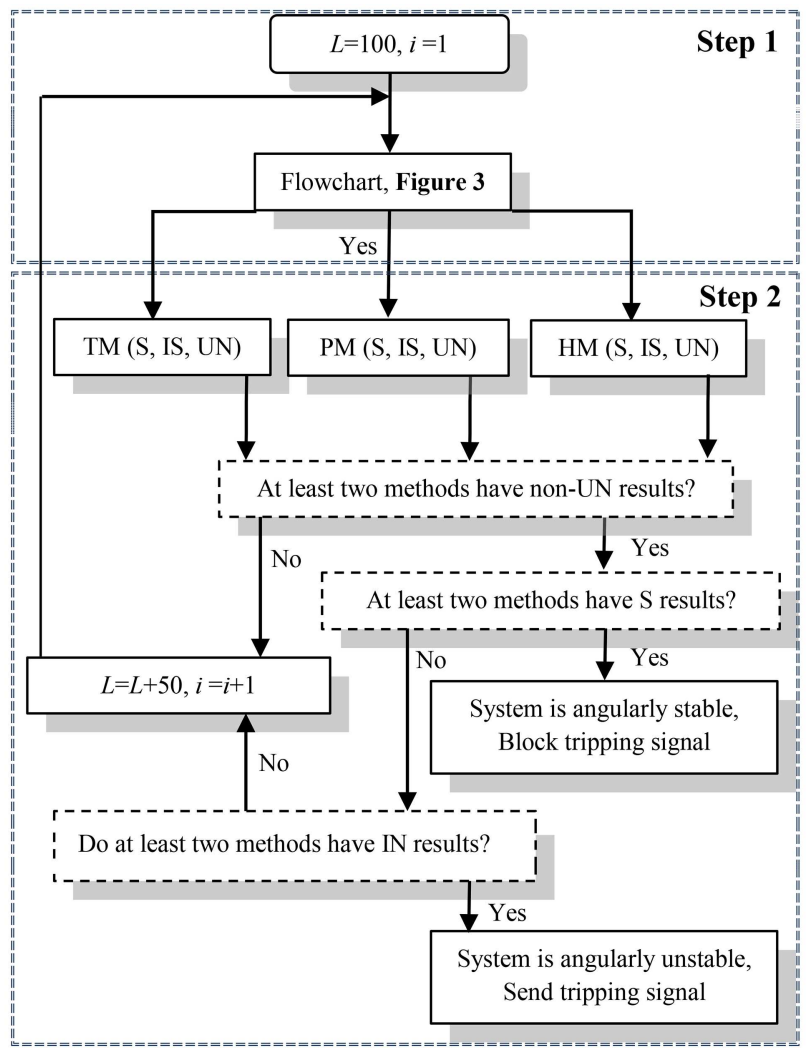

Figure 7. Flowchart of the proposed angular stability prediction using response-based Generation Rejection Scheme (GRS).

fault at $6 \%$ of Line $5-7$ at $145 \mathrm{MW}$ generation level of G2. In this case, the actual bus phase angle is marginally stable, while the results of all the three prediction models in the FDW framework are inappropriately unstable. Having applied the EDW method and the proposed GRS in step $1(\mathrm{DW}=\mathrm{FH}=100$ $\mathrm{ms}$ ), TM predicts the stability of the system; however, in PM and HM, none of the instability and stability conditions are satisfied. Therefore, GRS decisionmaking will be postponed to the next step of prediction by extending the length of DW to $50 \mathrm{~ms}$. In Step 2 $(\mathrm{DW}=\mathrm{FH}=150 \mathrm{~ms})$, TM and $\mathrm{HM}$ predict angular stability, while PM makes the opposite prediction.

For 2400 different case studies (2250 LLLG faults, 120 LLG faults, and 30 LG faults along different locations on Lines 7-5 and 7-8, with 100 and $120 \mathrm{~ms}$ fault durations at different generation levels of G2) consisting of 151 unstable cases and 2249 stable ones, the proposed GRS worked well in $96.03 \%$ of unstable scenarios (145 cases) and $96.93 \%$ of stable scenarios (2180 cases). In these simulation studies, the more severe the fault condition, the shorter the time to reach instability. In these circumstances, the speed of the GRS is very important in predicting the future behavior of generating unit's rotor angle trajectory. If the method works correctly, there will be an opportunity to open the GCB and prevent instability of the generating unit. Therefore, $T_{D}$ is considered the severity index. Meanwhile, the minimum value of $T_{D}$ (the most severe fault created) for the LLLG fault with a duration equal to $120 \mathrm{~ms}$ at $0.1 \%$ of Line $7-5$ at 155 MW generation level of G2 is $75 \mathrm{~ms}$. The maximum value of $T_{D}$ is $180 \mathrm{~ms}$ (the low-severity fault that causes the generating unit to be unstable) for the LLG fault at the $10 \%$ of Line $7-5$ for $151 \mathrm{MW}$ generation level of G2 and $100 \mathrm{~ms}$ fault duration, which is greater than $T_{A c t}$ of GCB. These performance metrics are remarkably higher than those associated with the existing method; however, they also prove that further research is still required in this area.

In real bulk power systems, the behavior of the generating unit will be different depending on the configuration of the lines connected to the power plant, the number of committed units, the frequency control status of each one of them, and the inertia of the whole power system. Meanwhile, the proposed GRS is applied to the real-world power plant which consists of four generating units, each rated at 312.5 MVA and $250 \mathrm{MW}$ [22]. Three real events are simulated based on [22] and the predicted transient stability behaviors are compared with the rotor angle measurements recorded by PMU. In all cases, the proposed GRS correctly predicted the behavior of the angular stability of the generating unit.

\section{Conclusions}

A response-based Generation Rejection Scheme (GRS) along with trigonometric, polynomial, and hybrid models of rotor angle trajectory was proposed in this 
paper based on Phasor Measurement Unit (PMU) measurement data. GRS as an emergency control system was designed to initiate a preplanned outage of accelerated generating units of power plants to take rapid corrective actions for preventing the rest of generating units from loss of synchronism. By applying the Forecast Horizon (FH) to the aforementioned prediction model, the accuracy of these models and the performance of the new GRS improved. The proposed models with/without FH were compared by actual responses based on the curve-fitting procedure through a comprehensive suite of simulations on the Single Machine Equivalent (SIME) and WSCC 9 bus test bed. Obtained results demonstrated that the FH had a significant impact on the accuracy of the proposed models and GRS performance. In addition, these models are response-oriented (independent of equipment models, system configuration, and generation level), enjoy simple flowcharts and less burdensome equations as well as very short prediction time, and are easy to use. Furthermore, according to the new fast communication media such as optic fiber, it is proposed that the feasibility of the proposed GRS and transient stability prediction methods for Wide-Area Measurement, Protection, And Control (WAMPAC) system be investigated.

\section{References}

1. Goh, H., Chen, K., Crocker, F., et al. "A review on equipment protection and system protection relay in power system", International Journal of Integrated Engineering, 9, pp. 7-12 (2017).

2. Shafeeque Ahmed, K., Prabhakar Karthikeya, Sh., and Sahoo, S. "Special protection schemes: A survey and vision for the future", Applied Mechanics and Materials, 839, pp. 49-53 (2016).

3. Pordanjani, I.R., Abyaneh, H.A., Sadeghi, S.H.H., et al. "Risk reduction in special protection systems by using an online method for transient instability prediction", International Journal of Electrical Power \& Energy Systems, 2, pp. 156-62 (2010).

4. Daham, M., Seog-Joo, K., Sangsoo, S., et al. "Computing safety margins of a generation rejection scheme: A framework for online implementation", IEEE Trans. Smart Grid, 9(3), pp. 2337-2346 (2018).

5. Karady, G.G. and Jun, G. "A hybrid method for generator tripping", IEEE Trans. Power Systems, 17, pp. 1102-1107 (2002).

6. Aminifar, F., Safdarian, A., Fotuhi-Firuzabad, M., et al. "A multi-objective framework for enhancing the reliability and minimizing the cost of PMU deployment in power systems", Scientia Iranica, 6, pp. 2917-2927 (2016).

7. Khandani, A. and Akbari Forou, A. "Providing transient stability by excitation system response improve- ment methods through long-term contracts", Scientia Iranica, 26(3), pp. 1652-1663 (2019).

8. Xiaochen, W., Jinquan, Z., Aidong, X., et al. "Review on transient stability prediction methods based on real time wide-area phasor measurements", Electric Utility Deregulation and Restructuring and Power Technologies (DRPT), 4th International Conference, pp. 320326 (2011).

9. Choi, D., Lee, S.H., Kang, Y.C., et al. "Analysis on special protection scheme of Korea electric power system by fully utilizing STATCOM in a generation side", IEEE Trans. Power Systems, 32(3), pp. 18821890 (2017).

10. Weckesser, T., Jóhannsson, H., and $\phi$ stergaard, J. "Real-time remedial action against aperiodic small signal rotor angle instability", IEEE Trans. Power Systems, 31(1), pp. 387-396 (2016).

11. Yang, H., Zhang, W., Shi, F., et al. "PMU-based model-free method for transient instability prediction and emergency generator-shedding control", International Journal of Electrical Power \& Energy Systems, 105, pp. 381-393 (2019).

12. Esmaili, M., Hajnoroozi, A.A., and Shayanfar, H.A. "Risk evaluation of online special protection systems", International Journal of Electrical Power \& Energy Systems, 41, pp. 137-144 (2012).

13. Liu, C.W. and Thorp, J. "Application of synchronised phasor measurements to real-time transient stability prediction", IEE Proceedings - Generation, Transmission and Distribution, 142, pp. 355-360 (1995).

14. Rajapakse, A.D., Gomez, F., Nanayakkara, K., et al. "Rotor angle instability prediction using postdisturbance voltage trajectories", IEEE Trans. Power Systems, 25(2), pp. 947-56 (2010).

15. Bretas, N.G. and Phadke, A.G. "Real time instability prediction through adaptive time series coefficients", Power Engineering Society Winter Meeting, IEEE, 1, pp. 731-736 (1999).

16. Salimian, M.R. and Aghamohammadi, M.R. "Intelligent out of step predictor for inter area oscillations using speed-acceleration criterion as a time matching for controlled islanding", IEEE Transactions on Smart Grid, 4, pp. 2488-2497 (2016).

17. Kamali, S., Amraee, T., and Bathaee, S.M.T. "Prediction of unplanned islanding using an energy based strategy", IET Generation, Transmission \& Distribution, 1, pp. 183-191 (2016).

18. Zima, M., Special Protection Schemes in Electric Power Systems, EEH-Power Systems Laboratory, pp. 1-22 (2002).

19. Weihui, F., Sanyi, Z., McCalley, J.D., et al. "Risk assessment for special protection systems", IEEE Trans. Power Systems, 17, pp. 63-72 (2002).

20. Kundur, P. Power System Stability \& Control, 5th Reprint, Tata McGraw-Hill, New Delhi (2005). 
21. Teimourzadeh, S., Davarpanah, M., Aminifar, F., et al. "An adaptive auto-reclosing scheme to preserve transient stability of microgrids", IEEE Trans. Smart Grid, 9(4), pp. 2638-2646 (2016).

22. Hajnoroozi, A.A., Aminifar, F., and Ayoubzadeh, H. "Generating unit model validation and calibration through synchrophasor measurements", IEEE Transactions on Smart Grid, 6, pp. 441-449 (2015).

23. DIgSILENT PowerFactory, available at:/http://www. digsilent.de/.

\section{Biographies}

Ali A. Hajnorouzi was born in Golpayegan, Iran, 1987. He received the BSc degree from the Isfahan University of Technology, Isfahan, Iran in 2009 and the MSc (Hons.) degree from the Iran University of Science and Technology, Tehran, Iran in 2011, both in Electrical Engineering. He is currently with Iran University of Science and Technology, Tehran as a PhD student. His current research interests include wide-area measurement systems, transient and voltage stability, special protection schemes, and model validation.

Farrokh Aminifar (SM'15) is currently an Associate Professor at the School of Electrical and Computer
Engineering, University of Tehran, Tehran, Iran. He has been at the Robert W. Galvin Center for Electricity Innovation, Illinois Institute of Technology, Chicago, IL, USA since 2009. He is the editor for the IEEE Transactions on Sustainability, IEEE Power Engineering Letters, and IET Smart Grid. He was the Guest Editor-in-Chief and Guest Editor of four special issues of the IEEE Transactions On Smart Grid. He was the recipient of the 2011 IEEE Iran Section Best PhD Dissertation Award, the 2013 IEEE/PSO Transactions Prize Paper Award, the 2015 IEEE Iran Section Young Investigator Award, and the 2017 Outstanding Young Scientist Award of Iran National Academy of Science. His current research interests include wide-area measurement systems, resilience modeling and assessment, and smart-grid technologies.

Heidar Ali Shayanfar received the BS and MSE degrees in Electrical Engineering in 1973 and 1979, respectively, and the $\mathrm{PhD}$ degree in the same field from Michigan State University, Lansing, MI, USA in 1981. He is currently a Full Professor at the Department of Electrical Engineering, Iran University of Science and Technology, Tehran, Iran. His current research focuses on the application of artificial intelligence to power systems, dynamic load modeling, voltage collapse, and smart grid. 